\title{
Temperature-Sensitive lonic Liquid-Based Dispersive Microextraction for Removal of Industrial Dyes in Water
}

\author{
Y. Wang $\dagger$, M.C. Wei, Q.C. Yu and M.T. Zhu \\ College of Life and Health Sciences, Anhui Science and Technology University, Fengyang 233100, P.R. China \\ $†$ Corresponding author: Y. Wang; yanwang0129@126.com
}

Nat. Env. \& Poll. Tech.

Website: www.neptjournal.com

Received: 19-01-2020

Revised: $18-02-2020$

Accepted: $16-04-2020$

\section{Key Words:}

Ionic liquid

Temperature-sensitive

Microextraction

Dye removal

\begin{abstract}
In this study, a new and effective temperature-sensitive ionic liquid-based dispersive microextraction method was developed for dye removal from water. Through the temperature change, the ionic liquid (IL) could complete dispersion in the aqueous phase to fully contact with the dyes, and then it was recovered as particles enriching target substance by cooling. Parameters including chemical structure and amount of IL, dispersing solvent and its volume, extraction temperature, extraction time, $\mathrm{pH}$ adjustment and ionic strength were investigated and optimized in detail. Under the optimized conditions, the extraction recovery was $98.9 \%$ of methyl violet, $95.6 \%$ of Congo red, and $98.8 \%$ of methylene blue, respectively. The results showed that the major driving force for the extraction might be the hydrophobic interaction between the dye and IL. In short, the proposed technique is rapid, simple, relatively inexpensive and effective. This implies meaningful information for further research and application in the field of actual samples.
\end{abstract}

\section{INTRODUCTION}

Currently, food and textile industries often directly discharge significant raw coloured sewage due to pigments and dyes. Most of the dyes containing nondegradable aromatic structure have been considered to be one of the most problematic wastewaters (Liu et al. 2014). Those dyes have been reported to be genotoxic (Salem 2000). So, the development of an effective, viable, simple and rapid method for treatment and disposal of hazardous dyes is urgently needed. Various techniques including adsorption, biological process and photo-degradation have been generally used (Jiang et al. 2011, Sun et al. 2011, Zhu et al. 2011). However, these methods are commonly time-consuming, high solvent consumption, costly or ineffective.

To minimize the extraction process, several techniques have been developed. Among them, dispersive liquid-liquid microextraction (DLLME) has attracted wide interest in separation field due to its rapidity and simplicity (Zhao et al. 2012). In this technique, the dispersive solvent is injected immediately into the sample solution. Then a cloudy solution possessing droplets of extraction solvent is shaped, and the hydrophobic target analytes are transferred to the extraction phase, followed by centrifugation or filtration for recovery. The exposed large surface between sample analytes and extraction solvent can result in rapid mass transfer and high extraction efficiency. Ionic liquids (ILs) are molten salts and exhibit unique physicochemical properties such as tunable viscosity and solubility, negligible vapour pressure, high thermal stability, and non-flammability (Rogers \& Seddon 2003). They have been recently proposed as extraction solvents instead of traditional organic solvents in DLLME procedure (Abdolmohammad-Zadeh \& Sadeghi 2010, Jha et al. 2018, Liu et al. 2009, Zhao et al. 2016a). Although abundant inspiring results have been realized, ILs still have some weaknesses in the DLLME procedure, including emulsification, relatively high viscosity and difficult recovery (Liu et al. 2014, Pena-Pereira et al. 2009). In recent years, temperature-sensitive ionic liquids, which can regulate their solubility in some solvents by changing the temperature, have got a great deal of attention. Temperature-sensitive ionic liquid-based dispersive liquid-liquid microextraction (TSILDLLME) is gradually becoming a new hot and promising technique (Padilla-Alonso et al. 2017, Sheikhian \& Shirafkan 2016, Zhang \& Shi 2010). In this process, the dispersion of IL can be achieved by heating, thus solubilizing IL in the sample solution to produce a homogeneous system. Then it was recovered as tiny particles enriching target solute by cooling. The special property makes it combine the advantages of both homogeneous and heterogeneous extractants.

Based on the above background, the present work aims to explore an effective way with solid hydrophobic IL (a kind of 
temperature-sensitive ionic liquids) in the proposal of dyeing water. In this work, several $N$-butylbenzothiazolium ILs were prepared, and the development of a TSIL-DLLME procedure to extract three dyes (Methyl violet, MV; Methylene blue, $\mathrm{MB}$; Congo red, CR) from water samples was proposed. All the variables, affecting the extraction process, were investigated and optimized in detail. The suggested method was proved to be available, rapid, simple and effective. The message acquired in this work provided an alternative approach for the removal of the dye in the practical application.

\section{MATERIALS AND METHODS}

\section{Reagents and Materials}

MV, CR and MB were purchased from Shanghai Macklin Biochemical Co., Ltd (China). 1-Bromobutane, 1-bromopentane, 1-bromohexane, 1-bromoheptane, and potassium hexafluorophosphate were of analytical grade and purchased from Aladdin Reagent Co. Ltd. (China). Benzothiazole was obtained from CS-Pharm Chemical Co. Ltd. (China). ILs, like $N$-butylbenzothiazolium hexafluorophosphate $\left(\left[\mathrm{C}_{4} \mathrm{Bth}\right]\right.$ $\left.\left[\mathrm{PF}_{6}\right]\right), N$-pentylbenzothiazolium hexafluorophosphate ([ $\left.\left.\mathrm{C}_{5} \mathrm{Bth}\right]\left[\mathrm{PF}_{6}\right]\right), N$-hexylbenzothiazolium hexafluorophosphate $\left(\left[\mathrm{C}_{6} \mathrm{Bth}\right]\left[\mathrm{PF}_{6}\right]\right)$ and $N$-heptentylbenzothiazolium hexafluorophosphate $\left(\left[\mathrm{C}_{7} \mathrm{Bth}\right]\left[\mathrm{PF}_{6}\right]\right)$ were synthesized as described by a reference (Liu et al. 2014). All other chemicals were of analytical grade or higher.

\section{Extraction Procedure}

$10.0 \mathrm{~mL}$ dye solution (40 mg/L) was poured into a $25 \mathrm{~mL}$ well-sealed colourimetric tube. $1 \mathrm{~mL}$ of dispersing solvent containing $100 \mathrm{mg}\left[\mathrm{C}_{\mathrm{n}} \mathrm{Bth}\right]\left[\mathrm{PF}_{6}\right](\mathrm{n}=4,5,6,7)$ was injected rapidly into the aqueous solution to form a cloudy solution. Then the mixture was heated at a controlled temperature to become a homogeneous system, and the analytes were extracted into the IL phase. Hereafter, the tube was cooled in an ice bath for 15 min to obtain a turbid solution. The

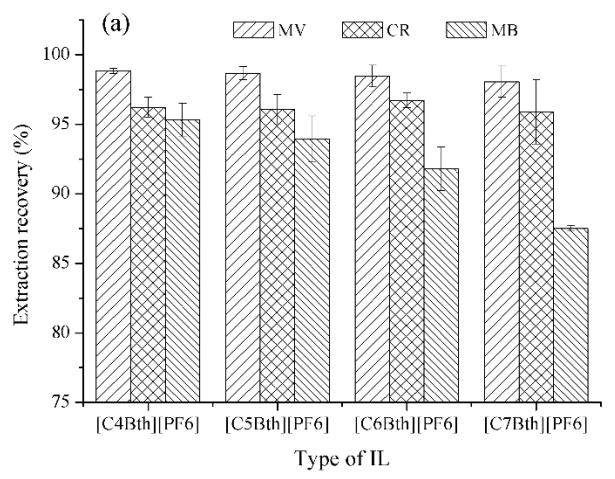

dispersed fine particles of the extraction phase were filtrated, and the concentration of the dye in the filtrate was subject to the quantitative analysis on a UV-vis spectrophotometer. All experiments were performed in triplicate.

The extraction recovery $(E R, \%)$ was defined as the percentage of the total dye amount extracted to the sedimented phase:

$$
E R(\%)=\left(1-\frac{C_{f} \times V_{f}}{C_{0} \times V_{0}}\right) \times 100
$$

Where, $C_{f}$ and $C_{0}$ are the dye concentration $(\mathrm{mg} / \mathrm{L})$ in the filtrate phase and the sample solution, $V_{f}$ and $V_{0}$ correspond to the volume of the filtrate solution and the sample solution, respectively.

\section{RESULTS AND DISCUSSION}

\section{Selection of IL and Dispersive Solvent}

An appropriate type of IL is essential for efficient extraction. In this work, four hydrophobic ILs were investigated, including $\left[\mathrm{C}_{4} \mathrm{Bth}\right]\left[\mathrm{PF}_{6}\right],\left[\mathrm{C}_{5} \mathrm{Bth}\right]\left[\mathrm{PF}_{6}\right],\left[\mathrm{C}_{6} \mathrm{Bth}\right]\left[\mathrm{PF}_{6}\right]$, and $\left[\mathrm{C}_{7} \mathrm{Bth}\right]$ $\left[\mathrm{PF}_{6}\right]$. As can be observed, $\left[\mathrm{C}_{4} \mathrm{Bth}\right]\left[\mathrm{PF}_{6}\right]$ formed the best dispersion cloud during the cooling step. The phenomenon may be due to the relatively lower solubility of $\left[\mathrm{C}_{4} \mathrm{Bth}\right]$ $\left[\mathrm{PF}_{6}\right]$ in the system. Furthermore, the dyes showed a better affinity for $\left[\mathrm{C}_{4} \mathrm{Bth}\right]\left[\mathrm{PF}_{6}\right]$. The results were shown in Fig.1a. Considering these results, $\left[\mathrm{C}_{4} \mathrm{Bth}\right]\left[\mathrm{PF}_{6}\right]$ was chosen as the most appropriate extraction solvent for further experiments.

The dispersive solvent should effectively disperse the IL into the sample solution to increase the contact area. And the main point to be concerned in selecting dispersive solvent is its miscibility in both sample solution and IL. For this purpose, methanol, ethanol and acetonitrile were preliminarily screened and then tested as potential dispersive solvents. The results, as shown in Fig.1b, demonstrated that acetonitrile exhibited the highest extraction efficiency for the three target dyes. Thus, acetonitrile was chosen as the disperser solvent for further study.

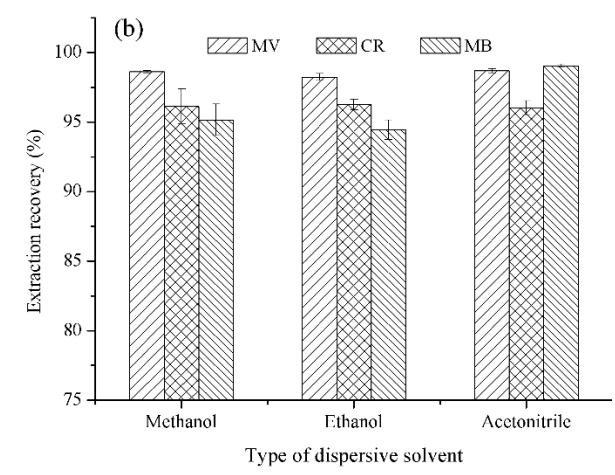

Fig. 1: Effects of various ILs and dispersive solvents on extraction recovery. 


\section{Effect of the Amount of IL}

To establish the optimum amount of IL, the effect of the amount of $\left[\mathrm{C}_{4} \mathrm{Bth}\right]\left[\mathrm{PF}_{6}\right]$ was investigated in the range of 20 $120 \mathrm{mg}$. This parameter was important as a very low amount of extraction solvent could complicate the recovery of the sedimented phase, while a very high amount needed much time to disperse the IL into the sample solution, which will affect the mass transfer. As can be observed in Fig.2, higher extraction recovery was obtained by increasing IL amount to $50 \mathrm{mg}$. Further increasing the IL amount showed no obvious change. The results indicated that $50 \mathrm{mg}$ of IL was sufficient to extract dyes in the proposed system. Thus, $50 \mathrm{mg}$ of IL was used for the following experiments.

\section{Effect of Extraction Temperature}

Temperature acts an important role for the complete dispersion of IL and mass transfer of dyes, which can increase the contact area between the dyes and IL (Zhang \& Shi 2010). To evaluate the effect of extraction temperature on the recoveries of dyes, different temperature ranging from 40 to $90^{\circ} \mathrm{C}$ were tested. As shown in Fig. 3, the extraction recovery enhanced gradually with the increasing temperature and then remained stable. The rising temperature could improve the solubility and dispersion of IL into the sample solution. However, a higher temperature might lead to a certain amount of IL dissolving in the sample solution, in turn, affect the generation of the cloudy solution after cooling (Zhang et al. 2012). Besides, whether at $60^{\circ} \mathrm{C}$ or $70^{\circ} \mathrm{C}$, there was no significant difference. Therefore, $60^{\circ} \mathrm{C}$ was selected as the further operating temperature.

\section{Effect of Extraction Time}

To study the effect of extraction time on the extraction recovery of dye into the IL phase, different extraction time ranging from 5 to $50 \mathrm{~min}$ were tested. As shown in Fig. 4, the maximum extraction recovery of dye into IL phase

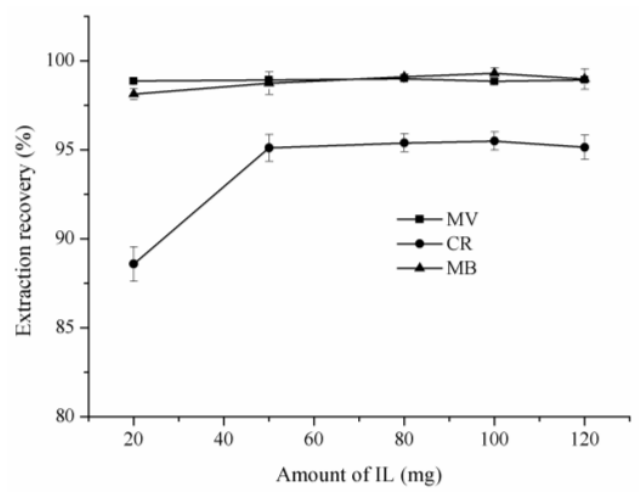

Fig. 2: Effect of amount of IL on extraction recovery. was related to the extraction time of $10 \mathrm{~min}$, and a longer time could not obtain higher extraction recovery. When the mixture of IL and the dispersive solvent was injected into the sample solution, it would take a short period of time for the dyes to transfer from aqueous phase to IL phase and to reach an equilibrium state. But the volatilization of acetonitrile might lead to the loss of dyes as the extraction time went longer. This behaviour was also observed by Liang et al. (2017), who summarized that with a longer time, the volatilization of organic solvent and consequent thermal effect of analytes could affect extraction efficiency. Thus, 10 min was selected as the optimum extraction time.

\section{Effect of Dispersive Solvent Volume}

The volume of acetonitrile affects the dispersion degree of $\left[\mathrm{C}_{4} \mathrm{Bth}\right]\left[\mathrm{PF}_{6}\right]$ in the aqueous phase, consequently influencing the formation of dispersion cloud, finally affecting the extraction efficiency. Therefore, the effect of the volume of acetonitrile was studied in the range of 0.5-1.5 mL. As shown in Fig.5, higher extraction recovery was obtained by increasing acetonitrile volume to $1.0 \mathrm{~mL}$, and then it slightly decreased as the volume increasing. At low volume, the cloudy solution of IL could not form completely. On the contrary, the amount of the sedimented phase and extraction recovery of target dyes would decrease due to the increased solubility of IL in the sample solution. Hence, $1.0 \mathrm{~mL}$ of acetonitrile was chosen as the optimum disperser volume.

\section{Effect of Sample pH}

The $\mathrm{pH}$ of the sample solution is an essential factor in the extraction process. It could affect the existing form of the targets and the interaction mechanism between targets and IL. In this work, a broad $\mathrm{pH}$ range of 4.0-10.0 was evaluated and the results were presented in Fig.6. It was clear that the extraction recovery of MV and MB had not a remarkable change in the investigated $\mathrm{pH}$ range, while it was not the case in CR solution. The recovery of CR increased and reached a

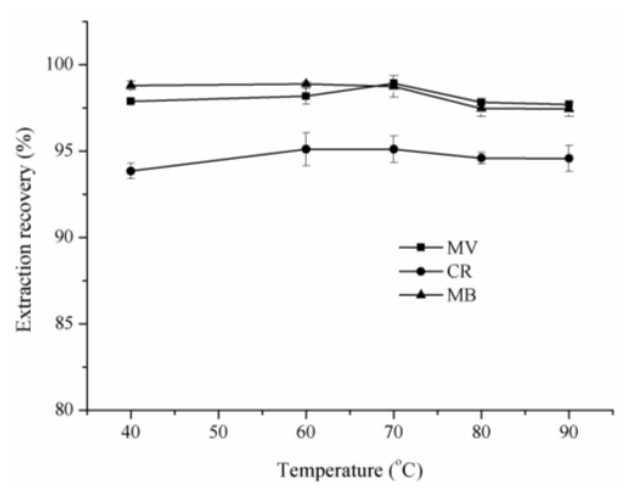

Fig. 3: Effect of extraction temperature on extraction recovery. 


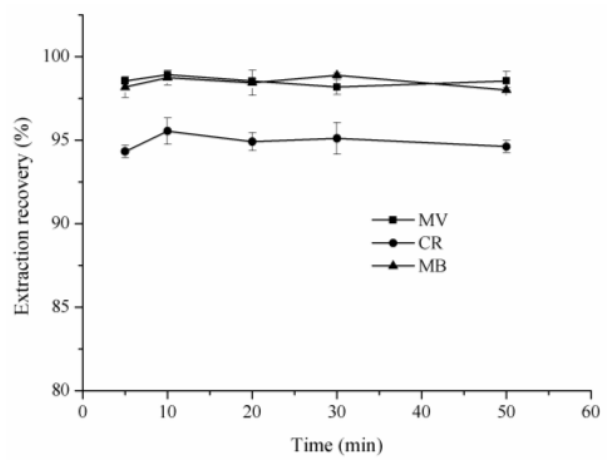

Fig. 4: Effect of extraction time on extraction recovery.

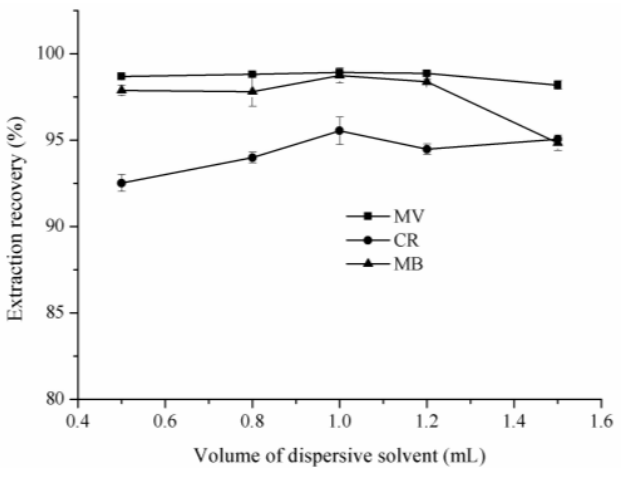

Fig. 5: Effect of dispersive solvent volume on extraction recovery.

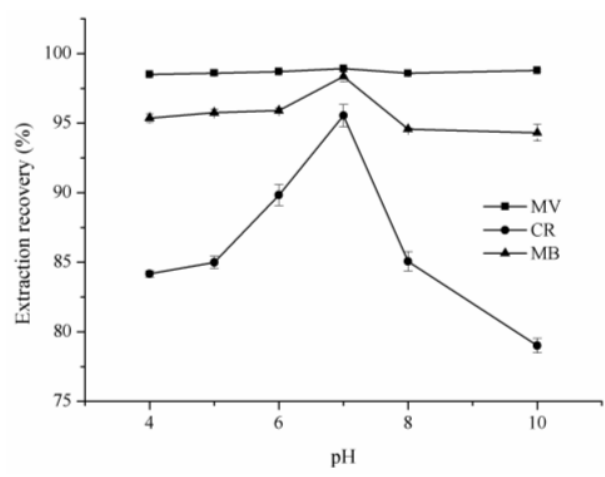

Fig. 6: Effect of sample $\mathrm{pH}$ on extraction recovery.

maximum value at a $\mathrm{pH}$ of 7.0, and thereafter decreased. The hydrophobic affinity of dye molecules to the IL phase may be driving force for extraction of dye into IL phase (Liu et al. 2014). Considering these results, the $\mathrm{pH}$ of 7.0 was chosen for the subsequent assay.

\section{Effect of Ionic Strength}

Under the previous optimum conditions, the effect of ionic strength was investigated by adding $\mathrm{NaCl}(0.2-1.0 \%, \mathrm{~g} / \mathrm{mL})$. Generally, the addition of salt could increase the extraction efficiency due to the possible salting-out effect. However, in the current study, the ionic strength did not have a remarkable effect on the extraction of dyes (data not shown in figure). This behaviour was also observed by Liang (Liang et al. 2017). Thus, this TSIL-DLLME procedure was performed without salt addition to the aqueous solution.

\section{Analysis of Real Water Samples}

To further evaluate the applicability and accuracy of the proposed method, drinking water and wastewater samples were analysed. To assess the matrix effect, water samples were spiked with target dyes at the concentration level of 10 $\mathrm{mg} / \mathrm{mL}(\mathrm{n}=3)$. Theoretically, the recovery values will not exceed $100 \%$ in the recovery experiment. However, inherent errors such as operation error and system error are inevitable in practice. Thus, the recovery values were higher than $100 \%$. It is a common phenomenon in method validation. In our study, the relative recovery values were in the range of 91.7$100.6 \%$, with RSD of $1.5-4.1 \%$. The results were summarized in Table 1. The results indicated that the suggested approach could be employed as an effective technique to extract these dyes from water.

Table 1: The relative recoveries and standard deviations of dyes in water samples.

\begin{tabular}{|llllll|}
\hline \multirow{2}{*}{ Analyte } & Added $(\mathrm{mg} / \mathrm{mL})$ & \multicolumn{2}{l|}{ Relative recovery $(\%)$} & & RSD $(\%)$ \\
\cline { 3 - 6 } & & drinking water & wastewater & drinking water & wastewater \\
\hline MV & 10.0 & 100.6 & 97.8 & 2.6 & 3.7 \\
CR & 10.0 & 93.0 & 91.7 & 1.8 & 4.1 \\
MB & 10.0 & 95.8 & 93.5 & 1.5 & 3.1 \\
\hline
\end{tabular}


Table 2: Comparison of TSIL-DLLME with other procedures for the removal of dyes.

\begin{tabular}{|c|c|c|c|c|c|}
\hline Target & Method & Extractant & $\begin{array}{l}\text { Extractant volume } \\
\text { or amount }\end{array}$ & $\begin{array}{l}\text { Recovery } \\
(\%)\end{array}$ & Reference \\
\hline $\begin{array}{l}\text { Malachite green, Crystal Violet, Methylene } \\
\text { blue }\end{array}$ & MSPE-DLLME & [Hpy]NTf2 & $70 \mu \mathrm{L}$ & $86.1-100.3$ & (Liang et al. 2017) \\
\hline Malachite green, Crystal violet, Methylene blue & SPE & MCAX cartridges & _- & 75 & (Xu et al. 2012) \\
\hline Malachite green, Crystal violet & IL-DLLME & {$\left[\mathrm{C}_{8} \mathrm{MIM}\right] \mathrm{PF}_{6}$} & $80 \mu \mathrm{L}$ & $91.7-97.2$ & (Zhang et al. 2012) \\
\hline $\begin{array}{l}\text { Malachite green, Gentian violet, Leucomala- } \\
\text { chite green, Leucogentian violet }\end{array}$ & MSPE & MWCNT@ $\mathrm{Fe}_{3} \mathrm{O}_{4}$ & $60 \mathrm{mg}$ & $87.0-92.8$ & (Zhao et al. 2016b) \\
\hline $\begin{array}{l}\text { Methyl violet, Congo red, } \\
\text { Methylene blue }\end{array}$ & TSIL-DLLME & {$\left[\mathrm{C}_{4} \mathrm{Bth}\right]\left[\mathrm{PF}_{6}\right]$} & $50 \mathrm{mg}$ & $91.7-100.6 \%$ & Present method \\
\hline
\end{tabular}

\section{Comparison of TSIL-DLLME Method with Previous Reported Procedures}

The extraction efficiencies of MV, CR and MB were notably enhanced by the presented TSIL-DLLME method. As summarized in Table 2, the extraction solvent using in DLLME is $\left[\mathrm{C}_{4} \mathrm{Bth}\right]\left[\mathrm{PF}_{6}\right]$, which involves some advantages such as temperature-sensitive phase-variable property, and reduction of exposure to toxic solvent. This method provided a relatively more sensitive and higher extraction recovery than previous reports. Meanwhile, the established method provided new insight into the extraction techniques of MV, $\mathrm{CR}$ and $\mathrm{MB}$ in water samples.

\section{CONCLUSION}

In this study, the TSIL-DLLME technique was successfully applied for the extraction of three dyes from water samples. The IL, $\left[\mathrm{C}_{4} \mathrm{Bth}\right]\left[\mathrm{PF}_{6}\right]$, was possible to effectively extract target dyes by only changing solution temperature, and could be recovered by simple filtration. Under the optimized conditions, the extraction recovery was $98.9 \%$ of MV, $95.6 \%$ of $\mathrm{CR}$, and $98.8 \%$ of $\mathrm{MB}$, respectively. The main driving force for the extraction was hydrophobic interaction between the dye and IL. In real water samples, the suggested technique also showed satisfactory performance. Compared with the conventional methods, the proposed temperature-sensitive IL-based dispersive extraction technique is simple, rapid, relatively inexpensive and effective. The results display a good alternative to the current methods for the extraction of dyes from aqueous solution.

\section{ACKNOWLEDGEMENTS}

The work was supported by the Talent Introduction Special Foundation of Anhui Science and Technology University (SKYJ201601).

\section{REFERENCES}

Abdolmohammad-Zadeh, H. and Sadeghi, G.H. 2010. Combination of ionic liquid-based dispersive liquid-liquid microextraction with stopped-flow spectrofluorometry for the preconcentration and determination of aluminium in natural waters, fruit juice and food samples. Talanta, 81(3): 778-785.

Jha, R.R., Singh, C., Pant, A.B. and Patel, D.K. 2018. Ionic liquid based ultrasound assisted dispersive liquid-liquid micro-extraction for simultaneous determination of 15 neurotransmitters in rat brain, plasma and cell samples. Anal. Chim. Acta., 1005: 43-53.

Jiang, R., Zhu, H.Y., Guan, Y.J., Fu, Y.Q., Xiao, L., Yuan, Q.Q. and Jiang, S.T. 2011. Effective decolorization of azo dye utilizing $\mathrm{SnO}_{2} / \mathrm{CuO} /$ polymer films under simulated solar light irradiation. Chem. Eng. Technol., 34(2): 179-185.

Liang, N., Hou, X., Huang, P., Jiang, C., Chen, L. and Zhao, L. 2017. Ionic liquid-based dispersive liquid-liquid microextraction combined with functionalized magnetic nanoparticle solid-phase extraction for determination of industrial dyes in water. Sci. Rep., 7: 13844.

Liu, J.B., Wang, L.T., Zhu, W.X., Jia, C.M., Deng, Q. and Yao, S. 2014. Temperature-assisted removal of triphenylmethane dyes from water with novel hydrophobic benzothiazolium ionic liquids. Sep. Sci. Technol., 49(1): 146-153.

Liu, Y., Zhao, E., Zhu, W., Gao, H. and Zhou, Z. 2009. Determination of four heterocyclic insecticides by ionic liquid dispersive liquidliquid microextraction in water samples. J. Chromatogr. A, 1216(6): 885-891.

Padilla-Alonso, D.J., Garza-Tapia, M., Chávez-Montes, A., GonzálezHorta, A., Waksman de Torres, N.H. and Castro-Ríos, R. 2017. New temperature-assisted ionic liquid-based dispersive liquid-liquid microextraction method for the determination of glyphosate and aminomethylphosphonic acid in water samples. J. Liq. Chromatogr. R. T., 40(3): 147-155.

Pena-Pereira, F., Lavilla, I. and Bendicho, C. 2009. Miniaturized preconcentration methods based on liquid-liquid extraction and their application in inorganic ultratrace analysis and speciation: A review. Spectrochim. Acta B, 64(1): 1-15.

Rogers, R.D. and Seddon, K.R. 2003. Ionic liquids-solvents of the future. Science, 302(5646): 792-793.

Salem, I.A. 2000. Kinetics of the oxidative color removal and degradation of bromophenol blue with hydrogen peroxide catalyzed by copper (II) supported alumina and zirconia. Appl. Catal. B-Environ., 28(34): $153-162$.

Sheikhian, L. and Shirafkan, M. 2016. Temperature-assisted ionic liquidbased dispersive liquid-liquid microextraction with following back- 
extraction for HPLCUV-Vis determination of 3-indole acetic acid in pea plants. J. Iran. Chem. Soc., 13(5): 903-911.

Sun, H., Wang, L., Qin, X. and Ge, X. 2011. Simultaneous determination of malachite green, enrofloxacin and ciprofloxacin in fish farming water and fish feed by liquid chromatography with solid-phase extraction. Environ. Monit. Assess., 179(1-4): 421-429.

Xu, Y.J., Tian, X.H., Zhang, X.Z., Gong, X.H., Liu, H.H., Zhang, H.J., Haung, H. and Zhang L.M. 2012. Simultaneous determination of malachite green, crystal violet, methylene blue and the metabolite residues in aquatic products by ultra-performance liquid chromatography with electrospray ionization tandem mass spectrometry. J. Chromatogr. Sci., 50(7): 591-597.

Zhang, H.F. and Shi, Y.P. 2010. Temperature-assisted ionic liquid dispersive liquid-liquid microextraction combined with high performance liquid chromatography for the determination of anthraquinones in Radix et Rhizoma Rhei samples. Talanta, 82(3): 1010-1016.

Zhang, Z., Zhou, K., Bu, Y., Shan, Z. Liu, J., Wu, X., Yang, L. and Chen, Z. 2012. Determination of malachite green and crystal violet in environmental water using temperature-controlled ionic liquid dispersive liquid-liquid microextraction coupled with high performance liquid chromatography. Anal. Methods, 4(2): 429-433.
Zhao, A., Wang, X., Ma, M., Wang, W., Sun, H., Yan, Z., Xu, Z. and Wang, H. 2012. Temperature-assisted ionic liquid dispersive liquid-liquid microextraction combined with high performance liquid chromatography for the determination of PCBs and PBDEs in water and urine samples. Microchim. Acta, 177(1-2): 229-236.

Zhao, J., Wei, D. and Yang, Y. 2016a. Magnetic solid-phase extraction for determination of the total malachite green, gentian violet and leucomalachite green, leucogentian violet in aquaculture water by high-performance liquid chromatography with fluorescence detection. J. Sep. Sci., 39(12): 2347-2355.

Zhao, J., Zhu, Y., Jiao, Y., Ning, J. and Yang, Y. 2016b. Ionic-liquid-based dispersive liquid-liquid microextraction combined with magnetic solid-phase extraction for the determination of aflatoxins B1, B2, G1, and G2 in animal feeds by high-performance liquid chromatography with fluorescence detection. J. Sep.Sci., 39(19): -3789 3797.

Zhu, H.Y., Fu, Y.Q., Jiang, R., Jiang, J.H., Xiao, L., Zeng, G.M., Zhao, S.L. and Wang, Y. 2011. Adsorption removal of congo red onto magnetic cellulose $/ \mathrm{Fe}_{3} \mathrm{O}_{4} /$ activated carbon composite: Equilibrium, kinetic and thermodynamic studies. Chem. Eng. J., 173(2): 494-502. 